\title{
Structural Identifiability: Tools and Applications
}

\author{
Torkel Glad, A. Sokolov \\ Division of Automatic Control \\ Department of Electrical Engineering \\ Linköpings universitet, SE-581 83 Linköping, Sweden \\ WWW: http://www. control.isy.liu.se \\ E-mail: torkel@isy.liu.se, @isy.liu.se
}

2nd December 2003

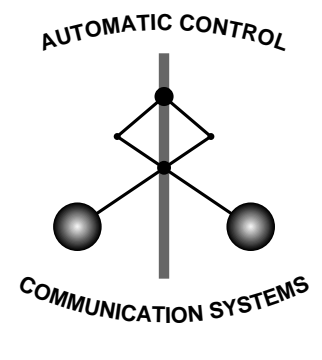

LINKÖPING

Report no.: LiTH-ISY-R-2557

Submitted to CDC'99

Technical reports from the Control \& Communication group in Linköping are available at http://www. control.isy.liu.se/publications. 


\begin{abstract}
The paper deals with the application of identifiability criteria to meanvalue models of turbocharged IC engines. A way of reducing such models to linear regressions using differential algebra is presented. The conditions of global identifiability and persistent excitation are formulated in explicit form for a given set of sensors. It is accompanied with a technique for reducing the set of sensors required for the engine identification. The software tools required are outlined and their complexity is discussed.
\end{abstract}

Keywords: Identifiability, differential algebra, internal combustion engine 


\section{Structural Identifiability: Tools and Applications}

\author{
T. Glad \\ Division of Automatic Control \\ Department of Electrical Engineering \\ Linköping University
}

\author{
A. Sokolov \\ Engine and Powertrain Electronics \\ Mecel AB \\ Box 73, SE-662 22 Åmål, Sweden
}

\section{Abstract}

The paper deals with the application of the identifiability criteria to mean-value models of turbocharged IC engines. A way of reducing such models to linear regressions using differential algebra is presented. The conditions of the global identifiability and the persistent excitation are formulated in explicit form for a given set of sensors. It is accompanied with the technique how to reduce the set of sensors required for the engine identification. The software tools required are outlined and their complexity is discussed.

\section{Parameterization of nonlinear models}

The models based on theoretical fundamentals such as physical, chemical, conservation and transport laws etc. are beneficial to improve the accuracy in description of phenomena. One the other hand, such models can be very complicated and control purposes require a semi-physical approach substituting some model parts by simplified heuristic relationships justified experimentally.

While conservation laws of dynamics are normally represented by bilinear differential-algebraic equations in its most general form, the statical physical and chemical relationships usually contain products of variables.

The relationships justified physically have a natural number of parameters which can be additionally reduced by mathematical methods using the engineering knowledge. The unknown static relationships are usually sought as formal mathematical expressions. As far as the set of parameters is specified the question of its identifiability [1] arises.

\section{Structural identifiability and choice of sensors}

The theoretical aspects of structural identifiability for differential-algebraic models are given in [1]. Structural identifiability depends on the sets of sensors, parameters and inputs.

The common problem faced by industries is how to reduce the number of sensors in a serial production unit without significant decrease in quality of the control system. Since most modern control systems are modelbased, the question of identifiability of model parameters is the question of our relying upon the given model structure and, hence, upon the control system in principle, foregoing identification itself.
In theory, if we are unlimited in the choice of sensors, most model structures will be identifiable. In practice, the set of measurements is very limited, especially on serial production units. Typically, various parts of the model are used as open loop estimation algorithms in the control strategy. During the control strategy calibration phase, the calibrator is, indeed, limited by the choice of sensors used in production comparing to the set used in the lab. Hence, the problem what minimal set of sensors should be used to identify a given model structure is an important one.

Thus, if one is faced by the problem of getting rid of the sensor measuring a variable $x(t)$ needed to estimate an important parameter $a$, one has to find a source of additional information. It can be obtained from the other measurements and model equations linking these measurements and their derivatives. An additional information source, common for all applications, is the prior knowledge. It often happens that one can know the range for a given parameter or its sign in advance.

Based on these sources the test can be performed whether the parameter $a$ is identifiable. If the answer is negative then one has to add one sensor, if positive then one can try to get rid of one of them and run the test again. In such an iterative way we will end up with the minimal set of sensors needed for a given model parameterization.

It turns out that this test can be carried out by differential algebra tools. It was shown in [1] that for the models described by differential-algebraic equations (DAE) there exists an explicit algorithm to test whether model free parameters can be uniquely recovered from the data. The algorithm does not use numeric data at all and, loosely speaking, reduces the identifiability problem to the following: whether the given model structure can be rearranged as a linear regression with respect to each parameter. The extensions of the algorithm have been developed in [2] taking into account the prior knowledge about the system, which can be written in the form of inequalities. Thus, the theoretical problem has been solved in an algorithmic form. The question left is the computational complexity of Ritt's algorithm which is the base of the technique [9].

In the paper given, the practical aspects of application of this algorithm to engine mean-value models are investigated. 


\section{Differential-algebraic representation of nonlinear models}

It was shown in [4] that any system with elementary functions can be rewritten in DAE form, i.e. consisting of polynomial equations in variables and their derivatives.

On the other hand, the first principles, used for the engine modeling, are originally of DAE type: the conservation of energy and mass, the gas dynamic relationships. Thus, the non-algebraic constructions are apparently introduced by model developers trying to rearrange equations in a simpler form eliminating the variables. In terms of identification such simplification is not desirable and DAE form of the model is preferable.

Differential algebra deals with systems described by polynomial equations $f(x, y, u)=0$ in which derivatives of variables are also admitted [9].

The differential algebra allows to determine for each system of DAE a characteristic set of equations which inherits the main properties of the original system and in most cases contains input-output relationship. There exists an algorithm, named Ritt's algorithm, constructing such characteristic set and obtaining an inputoutput relationship. Its computer implementation in [9] is the main tool used in this paper.

Let us consider an input-output relation $A(u, y)$ involving inputs $u$ and outputs $y$. Let the parameters be included in the coefficients $h_{k, j}$. A can be written in the form

$$
A(u, y)=I(u, y)\left(y_{k}^{\left(\nu_{k}\right)}\right)^{f_{k}}+\sum_{j=1}^{n_{k}} h_{k, j} \phi_{k, j}(u, y)
$$

where $I(u, y)$ is the leader of $A_{k}[1]$ and $f_{k}$ is the highest power of the term $y_{k}^{\left(\nu_{k}\right)}$.The terms $\phi_{k, j}(u, y)$ are expressions of the form:

$$
\phi_{k, j}(u, y)=y_{1}^{\alpha_{k, 0}} \dot{y}_{1}^{\alpha_{k, 1}} \cdots\left(u_{m}^{\left(\mu_{k, m}\right)}\right)^{\beta_{k, \mu_{k, m}}}
$$

, i.e. power products of the $u_{i}, y_{i}$ and their derivatives.

Definition 4.1 A canonical parameterization of the input-output relationship is one where the new parameters $\theta_{i}$ are chosen as the coefficients $h_{k, j}$ in (1).

Proposition 4.1 A canonical parameterization is globally identifiable for generic solutions $u, y$. Generic means "sufficiently general".

Proposition 4.2 The determinants

$$
D_{k}=\left|\begin{array}{ccc}
\phi_{k, 1} & \ldots & \phi_{k, n_{k}} \\
\phi_{k, 1}^{\prime} & \ldots & \phi_{k, n_{k}}^{\prime} \\
\vdots & & \vdots \\
\phi_{k, 1}^{\left(n_{k}-1\right)} & \ldots & \phi_{k, n_{k}}^{\left(n_{k}-1\right)}
\end{array}\right|
$$

are nonsingular, evaluated for a generic solution. Particular solutions satisfied to such conditions are called persistently excited. See proofs in [10].

\section{Engine model parameterization}

In the paper given, a basically configurated turbocharged engine is considered. For simplicity the submodels of fuel dynamics, combustion, intercooler and EGR system are not considered. The main modeling sources used are $[6,7]$. In this section the list of modeling equations is given. The more detailed description of the model is in [12].

\subsection{Turbocharger}

The dynamics of the turbocharger shaft is described by the energy conservation law:

$$
\frac{\partial}{\partial t} N_{t c}(t) N_{t c}(t)=\eta_{m} P_{t}(t)-P_{c}(t)
$$

where $\eta_{m}$ is the turbine mechanical efficiency and $N_{t c}$ is the shaft speed. The power $P_{t}(t)$ generated by turbine and the power $P_{c}(t)$ consumed by compressor can derived from the steady form of energy conservation:

$$
\begin{aligned}
& \frac{\partial}{\partial t} E_{c}(t)=0=P_{c}(t)+\left(T_{a m b}-T_{c}(t)\right) w_{c} C_{p} \\
& \frac{\partial}{\partial t} E_{t}(t)=0=-P_{t}(t)+\left(T_{e m}(t)-T_{a m b}\right) w_{t} C_{p}(5)
\end{aligned}
$$

The outlet temperature and the pressure in the turbocharger are assumed equal to the ambient constants $T_{a m b}, p_{a m b} ; T_{e m}, p_{e m}$ stand for the exhaust manifold temperature and pressure; $T_{c}, p_{c}$ are the compressor outlet temperature and pressure; $w_{c}, w_{t}$ are the mass flow rates through the compressor and the turbine, respectively. The compressor and turbine isentropic efficiencies $\eta_{c}, \eta_{t}$ are usually introduced by the relationships:

$$
\begin{aligned}
\eta_{c}(t)\left(\frac{T_{c}(t)}{T_{a m b}}-1\right) & =\left(\frac{p_{c}(t)}{p_{a m b}}\right)^{\frac{\gamma-1}{\gamma}}-1 \\
\left(\frac{T_{a m b}}{T_{e m}(t)}-1\right) & =\eta_{t}\left(\left(\frac{p_{a m b}}{p_{e m}(t)}\right)^{\frac{\gamma-1}{\gamma}}-1\right)
\end{aligned}
$$

The turbine efficiency is assumed constant and the compressor efficiency is assumed the polynomial:

$$
\eta_{c}=a_{4}+a_{5} N_{t c}+a_{6} N_{t c}^{2}+a_{7} w_{c}+a_{8} w_{c}^{2}+a_{9} w_{c} N_{t c}
$$

There are many other functional forms studied in the literature. It is taken here as a demonstration example. Denote by $a_{\eta_{c}}$ the parameter set of this polynomial.

The turbine mass air flow can be modeled with use of the orifice equation in quazi-isentropic conditions [12]:

$$
\frac{w_{t} \sqrt{R T_{e m}}}{p_{e m}}=\frac{A_{t} \frac{p_{a m b}}{p_{e m}} \sqrt{\frac{2 \eta_{t} \gamma\left(1-\left(\frac{p_{a m b}}{p_{e m}}\right)^{\left(\frac{\gamma-1}{\gamma}\right)}\right)}{\gamma-1}}}{1+\eta\left(\left(\frac{p_{a m b}}{p_{e m}}\right)^{\left(\frac{\gamma-1}{\gamma}\right)}-1\right)}
$$


where $A_{t}$ is the turbine orifice effective area which is assumed constant. No flow chocked or reversal is admitted during the experiments. The general set of parameters which is subject to identification for the turbocharger is given as follows:

$$
\mathcal{P}_{t c}=\left\{J_{t c}, \eta_{t}, \eta_{m}, A_{t}\right\} \cup\left\{a_{\eta_{c}}\right\}
$$

\subsection{Air Throttle}

The air throttle mass flow rate is usually modeled as the isentropic flow through the orifice $(\eta=1)$.

$$
\frac{w_{c} \sqrt{R T_{c}}}{p_{c}}=A_{a t} \sqrt{\frac{2 \gamma}{\gamma-1}\left(\left(\frac{p_{i m}}{p_{c}}\right)^{\frac{2}{\gamma}}-\left(\frac{p_{i m}}{p_{c}}\right)^{\frac{\gamma+1}{\gamma}}\right)}
$$

where $p_{i m}, T_{i m}$ are the pressure and temperature in the intake manifold. Again it is assumed that reversal and chocked flows are not admitted.

The effective flow area $A_{a t}(t)$ can be modeled by the following equation $[6,5]$ :

$$
A_{a t}(t)=a_{0}\left(1-\cos \left(a_{1} \alpha(t)+a_{2}\right)\right)+a_{3}
$$

The parameters $a_{i}$ has technical meaning and, hence, the prior knowledge can be formulated as a set of inequalities: $I_{a t}=\left\{a_{0} \geq 0, a_{1} \geq 0, a_{3} \geq 0\right\}$ The set of throttle parameters is summarized as:

$$
\mathcal{P}_{a t}=\left\{a_{0}, a_{1}, a_{2}, a_{3}\right\}
$$

\subsection{Manifolds}

One of the simplest ways to model the manifolds dynamics is to apply the mass conservation principle in conjunction with the ideal gas law [7] :

$$
\begin{aligned}
\frac{\partial}{\partial t} m_{i m}(t) & =w_{c}(t)-w_{b e}(t) \\
p_{i m}(t) & =\frac{m_{i m}(t)}{V_{i m}} R T_{i m}(t) \\
\frac{\partial}{\partial t} m_{e m}(t) & =w_{b e}(t)+w_{f}(t)-w_{t}(t) \\
p_{e m}(t) & =\frac{m_{e m}(t)}{V_{e m}} R T_{e m}(t)
\end{aligned}
$$

The mass air flow $w_{b e}$ to the combustion chamber is modeled by the following equation:

$$
w_{b e}(t) R T_{i m}(t)=\frac{\eta_{v o l}(t) N_{b e}(t) p_{i m}(t) V_{b e}}{120}
$$

where the function $\eta_{v o l}(t)$ is called the volumetric efficiency, $V_{b e}$ is the cylinder displacement volume. The volumetric efficiency assumed to be a polynomial [6] with the parameter set $a_{\eta_{v o l}}$ :

$$
\eta_{v o l}=a_{27}+a_{28} N_{b e}+a_{29} N_{b e}^{2}+a_{30} N_{b e} p_{i m} .
$$

Here $\mathcal{P}_{\text {im }} \cap \mathcal{P}_{\text {em }}=\left\{V_{i m}, a_{\eta_{\text {vol }}}\right\}$.

\subsection{Basic Engine}

The dynamics of the engine shaft can be described using the energy conservation law:

$$
\frac{\partial}{\partial t} N_{b e}(t) N_{b e}(t)=P_{b e}(t)-P_{l o s s}(t)-\mathcal{T}_{\text {load }}(t) N_{b e}(t)
$$

where $N_{b e}(t)$ is the engine shaft speed. Here $P_{b e}(t)$ is the power generated by the shaft, $P_{\text {loss }}(t)$ is the total loss power and $\mathcal{T}_{\text {load }}(t)$ is the load torque scaled with $\frac{30}{\pi}$.

The model of $P_{b e}(t)$ is taken for the spark-ignited engine [7] assuming $\lambda=1$ and ignoring the term for the spark advance:

$$
\begin{aligned}
P_{b e} & =w_{f} P_{P_{i m}}\left(a_{19}-a_{20} e^{-N_{b e} a_{21}}\right) . \\
P_{P_{i m}} & =\left(a_{16}+a_{17} p_{i m}+a_{18} p_{i m}^{2}\right)
\end{aligned}
$$

where $P_{P_{i m}}$ is the part dependent on the intake pressure. The set of parameters in this relationship is denoted by $a_{P_{b e}}$ and $a_{P_{P_{i m}}}$, respectively.

The total loss power, referring to the same source [7], is modeled by a quadratic polynomial in the engine speed $N_{b e}$ and the intake pressure $p_{i m}$ with the coefficients $a_{P_{\text {loss }}}$ :

$$
\begin{gathered}
P_{\text {loss }}=a_{22} N_{b e}+a_{23} N_{b e}^{2} \\
+a_{24} N_{b e}^{3}+a_{25} p_{i m} N_{b e}+a_{26} p_{i m} N_{b e}^{2} \\
\mathcal{P}_{b e}=\left\{J_{b e}, a_{P_{b e}}, a_{P_{l o s s}}, a_{P_{P_{i m}}}\right\}
\end{gathered}
$$

The equation system described in this section will be denoted shortly $\mathcal{E}$ in the rest of the paper.

\section{Nonlinear engine model as a cascade of linear regressions}

Definition 6.1 Let $\mathcal{M}$ be the set of measurements, $\mathcal{P}$ be the set of parameters. The system of equations $\mathcal{E}$ is called a linear regression in $\mathcal{P}$ with respect to $\mathcal{M}$ if it appears as a system of polynomial equations of the first order in arguments from $\mathcal{P}$ with the coefficients being known functions in the elements of $\mathcal{M}$ and their derivatives. These coefficients are called regressors.

If a model is rearranged as a linear regression in parameters then the identification of these parameters can be performed by well-developed and reliable least-squares techniques [3]. It will allow to bypass the local minima problem usually arising in the non-convex optimization.

Assume the ideal situation when we have a full set of measurements:

$$
\begin{array}{r}
\mathcal{M}_{i d}=\left\{N_{t c}(t), N_{\text {be }}(t), \mathcal{T}_{\text {load }}(t), p_{i m}(t), p_{\text {em }}(t), p_{c}(t),\right. \\
\left.T_{i m}(t), T_{e m}(t), T_{c}, w_{f}(t), \alpha(t)\right\}
\end{array}
$$

The general set of parameters is summarized as:

$$
\mathcal{P}=\mathcal{P}_{t c} \cup \mathcal{P}_{a t} \cup \mathcal{P}_{i m} \cup \mathcal{P}_{\text {em }} \cup \mathcal{P}_{b e}
$$


Proposition 6.1 There exists a transformation $F$ to the new parameter set $\Theta=F(\mathcal{P})$ such that the complete engine model $\mathcal{E}$ can be rearranged as a linear regression in $\Theta$ with respect to $\mathcal{M}_{i d}$

Generally, the proof of this proposition follows from the results of section 4 . The proof given below consists of four assertions. It is of constructive nature and its main steps consist of sequential conversion of engine subsystems to DAE form where it is necessary and application of Ritt's algorithm to generate input-output relationships.

Proposition 6.2 The air throttle model (10)-(11) can be rearranged to the following linear regression in the set of parameters

$$
\Theta_{a t}=\left\{\theta_{1}^{a t}, \theta_{2}^{a t}, \theta_{3}^{a t}, \theta_{4}^{a t}\right\}
$$

with respect to $\mathcal{M}_{i d}$ :

$$
\begin{aligned}
& y_{1}^{a t}(t)=\theta_{1}^{a t} \phi_{1}^{a t}(t)+\theta_{1}^{a t} \phi_{2}^{a t}(t)+\theta_{2}^{a t} \phi_{3}^{a t}(t) \\
& y_{2}^{a t}(t)=\theta_{4}^{a t} \phi_{4}^{a t}(t)
\end{aligned}
$$

where $\phi_{j}^{a t}, y_{i}^{a t}$ are the regressors with respect to $\mathcal{M}_{i d}$, $\theta_{j}^{a t}$ are new parameters and $i=1 \cdots 2, j=1 \cdots 4$ :

$$
\begin{aligned}
\theta_{1}^{a t} & =a_{0}{ }^{2} a_{1}{ }^{2}-a_{0}{ }^{2} a_{3}{ }^{2} a_{1}{ }^{2} \\
\theta_{2}^{a t} & =a_{0} a_{3} a_{1}{ }^{2} \\
\theta_{3}^{a t} & =a_{1}{ }^{2} \\
\theta_{4}^{a t} & =a_{2}
\end{aligned}
$$

The explicit form of the regressors here and below is given in [12].

Remark 6.1 The regressor $y_{2}^{a t}$ depends on the parameters from the first regression equation, i.e. the regressions form a cascade and the dependent regressors become known by consequent computations. Thus, it does not contradict to the definition of linear regression given in this section.

Remark 6.2 The practical implementation of the regressors generated can be done by sampling the derivatives involved. Notice, that the regressors contain only first derivatives of the measurement signals from $\mathcal{M}_{i d}$.

Remark 6.3 Most of the mathematical manipulations needed in this derivation are automatized [1, 2] with computer algebra systems. Below, we give an only manual proof to elucidate some steps showing how it works.

Proof of Proposition 6.2 By introducing new variables $z_{1}(t)=\cos \left(a_{1} \alpha(t)+a_{2}\right)$ and $z_{1}(t)=$ $\sin \left(a_{1} \alpha(t)+a_{2}\right)$, differentiating it and taking into account properties of trigonometric functions we obtain that the effective area equation (11) is equivalent to the following differential-algebraic system:

$$
\begin{aligned}
A_{a t}(t)-a_{0} z_{1}(t)-a_{0} a_{3} & =0 \\
\frac{d}{d t} z_{1}(t)+z_{2}(t) a_{1} \frac{d}{d t} \alpha(t) & =0 \\
\left(z_{1}(t)\right)^{2}+\left(z_{2}(t)\right)^{2}-1 & =0
\end{aligned}
$$

plus the equation

$$
\arccos \left(\frac{A_{a t}(t)-a_{3}}{a_{0}}\right)=a_{1} \alpha(t)+a_{2}
$$

Eliminating the variables $z_{1}, z_{2}$ by differentiation we obtain the equation:

$$
\begin{gathered}
\left(\frac{d}{d t} A_{a t}(t)\right)^{2}=a_{0}{ }^{2} a_{1}{ }^{2}\left(\frac{d}{d t} \alpha(t)\right)^{2} \\
-a_{1}{ }^{2}\left(\frac{d}{d t} \alpha(t)\right)^{2}\left(A_{a t}(t)\right)^{2}+2 a_{0} A_{a t}(t) a_{1}{ }^{2}\left(\frac{d}{d t} \alpha(t)\right)^{2} a_{3} \\
-a_{0}{ }^{2} a_{3}{ }^{2} a_{1}{ }^{2}\left(\frac{d}{d t} \alpha(t)\right)^{2}
\end{gathered}
$$

Taking into account the notations used we immediately obtain the first equation (21). The parameters $\theta_{0}^{a t}, \theta_{1}^{a t}, \theta_{3}^{a t}$ can then be determined by any relevant estimation procedure [3] if the excitation input $\alpha(t)$ is correctly chosen. The analysis of the algebraic system (22) shows that this system is uniquely resolvable with respect to the parameters $a_{a t}$ if we utilize the prior knowledge formalized in the inequality set $I_{a t}$. Substituting the parameters obtained in (24) we realize that this equation is also a linear regression in $a_{2}$. The assertion follows.

Proposition 6.3 The turbocharger model (3)-(9) can be rearranged as the following linear regression in the set of parameters:

$$
\Theta_{t c}=\left\{\theta_{1}^{t c}, \theta_{2}^{t c}, \theta_{3}^{t c}, \theta_{4}^{t c}, \theta_{\eta_{c}}, \theta_{p_{c}}\right\}
$$

with respect to $\mathcal{M}_{i d}$ :

$$
\begin{aligned}
y_{1}^{t c}(t) & =\theta_{1}^{t c} \phi_{1}^{t c}(t)+\theta_{2}^{t c} \phi_{2}^{t c}(t) \\
y_{2}^{t c}(t) & =\theta_{3}^{t c} \phi_{3}^{t c}(t) \\
y_{\eta_{c}}^{t c}(t) & =\theta_{\eta_{c}}^{t c} \phi_{\eta_{c}}^{t c}(t)
\end{aligned}
$$

where $\theta_{1}^{t c}=J_{t c}, \theta_{2}^{t c}=\eta_{m} A_{t}, \theta_{3}^{t c}=\eta_{t}, \theta_{\eta_{c}}^{t c}=a_{\eta_{c}}$.

The regressor vector $\phi_{\eta_{c}}^{t c}(t)$ is composed from the regressors of the compressor map. Since the turbocharger speed is known, no conversion to DAE form is required. The results follow directly rewriting the system (3)-(9). The parameters are to be computed recursively.

To represent the basic engine subsystem in compact regression form we make the additional assumptions that the loss power $P_{l o s s}(t)$ and the intake pressure dependent term denoted by $P_{p_{i m}}(t)$ in the expression (18) are estimated in advance and, hence, are known functions. Since they depend on the variables from $\mathcal{M}_{i d}$ one can attach them to this set as additional measurements.

$$
\tilde{\mathcal{P}}_{b e}=\left\{a_{19}, a_{20}, a_{21}, J_{b e}\right\}
$$

Proposition 6.4 The basic engine model (17)-(20) can be rearranged as the following linear regression in parameters

$$
\Theta_{b e}=\left\{\theta_{1}^{b e}, \theta_{2}^{b e}, \theta_{3}^{b e}, \theta_{4}^{b e}, \theta_{4}^{b e}\right\}
$$


with respect to $\tilde{\mathcal{M}}_{i d}=\mathcal{M}_{i d} \cup\left\{P_{\text {loss }}(t), P_{p_{\text {im }}}(t)\right\}$ :

$$
\begin{aligned}
& y_{1}^{b e}(t)=\theta_{1}^{b e} \phi_{1}^{b e}(t)+\theta_{1}^{b e} \phi_{2}^{b e}(t)+\theta_{3}^{b e} \phi_{3}^{b e}(t)+\theta_{4}^{b e} \phi_{4}^{b e}(t) \\
& y_{2}^{b e}(t)=\theta_{5}^{b e} \phi_{5}^{b e}(t)
\end{aligned}
$$
where $\theta_{1}^{b e}=J_{b e}, \theta_{2}^{b e}=a_{21}, \theta_{3}^{b e}=a_{19} a_{21}, \theta_{4}^{b e}=$
$J_{b e} a_{21}, \theta_{5}^{b e}=a_{20}$.

Proof of Proposition 6.4 Convert the system (17)(20) to DAE form. A new variable $z(t)$ and differential equation will appear. Apply Ritt's algorithm to this DAE system specifying the next inputs for elimination: $z(t), E_{b e}(t), P_{b e}(t)$. The resulting relationship does not contain the parameter $a_{20}$. That means that this parameter is not identifiable in this DAE form. However, it can be recovered from the original non-algebraic equation. Again, similar to air throttle model, we have the cascade construction of linear regressions.

Proposition 6.5 The manifold models $(12$, , 16) can be rearranged to the following linear regression in parameters $\Theta_{i m}=\left\{\theta_{1}^{i m}, \theta_{2}^{i m}, \theta_{3}^{i m}, \theta_{4}^{i m}, \theta_{5}^{i m}\right\}$ and $\Theta_{e m}=$ $\left\{\theta_{1}^{e m}, \theta_{2}^{e m}\right\}$ with respect to $\mathcal{M}_{i d}$ :

$$
\begin{array}{r}
y^{i m}(t)=\theta_{1}^{i m} \phi_{1}^{i m}(t)+\theta_{2}^{i m} \phi_{2}^{i m}(t)+\theta_{3}^{i m} \phi_{3}^{i m}(t)+ \\
\theta_{4}^{i m} \phi_{4}^{i m}(t)+\theta_{5}^{i m} \phi_{5}^{i m}(t) \\
y^{e m}(t)=\theta_{1}^{e m} \phi_{1}^{e m}(t)+\theta_{2}^{e m} \phi_{2}^{e m}(t)
\end{array}
$$

where $\theta_{1}^{i m}=V_{i m}, \theta_{2}^{i m}=a_{27}, \theta_{3}^{i m}=a_{28}, \theta_{4}^{i m}=$ $a_{29}, \theta_{5}^{i m}=a_{30}, \theta_{1}^{e m}=V_{e m}, \theta_{2}^{e m}=A_{t}$.

\section{Identifiability criteria}

This section deals with the criteria of identifiability for the model parameterization (3)-(20). It is shown that this model is globally identifiable with respect to the measurement set $\tilde{\mathcal{M}}_{i d}$. The condition of global identifiability, when the sensor of turbocharger speed is eliminated, is given. It is discussed how to choose the persistently exciting model inputs to perform engine identification.

The definition of global identifiability is given according to [1] in a modified form as follows:

Definition 7.1 $A$ model structure of equation, measurement and parameter set $\mathcal{E}, \mathcal{M}=\{z(t)\}, \mathcal{P}=\{\theta\}$ with prior knowledge formalized in the form of inequalities $I$ is globally identifiable if for any feasible $\theta^{\star}$ the following implication holds:

$$
z(t, \theta)=z\left(t, \theta^{\star}\right) \Rightarrow \theta=\theta^{\star} .
$$

If it holds in an open neighborhood of $\theta^{\star}$ then the model structure is called locally identifiable in $\theta^{\star}$.

Proposition 7.1 The engine model (3)-(20) with prior knowledge $I=I_{a t}$ is globally identifiable with respect to the parameter set $\mathcal{P}$ and measurement set $\tilde{\mathcal{M}}_{i d}$.
Proof of Proposition 7.1 The linear regressions derived in the propositions $6.2-6.5$ are canonically parameterized by construction. According to the results from section 4 they are globally identifiable. Hence, the identifiability is reduced to the question whether the systems of algebraic equations defined in the assertions of the section 6 and called here as identifiability tables are uniquely resolvable with respect to the original physical parameters. The identifiability table for the manifolds is trivially uniquely resolvable with respect to $\mathcal{P}_{i m} \cup \mathcal{P}_{e m} \cup\left\{A_{t}\right\}$. As a bonus we obtain the parameter $A_{t}$ usually referred to the turbocharger subsystem. With such a knowledge the identifiability table for turbocharger will be uniquely resolvable with respect to $\mathcal{P}_{t c}$. The identifiability table for the air throttle. is generally not resolvable uniquely with respect to $\mathcal{P}_{a t}$ but with the additional knowledge $I_{t c}$ it is. The basic engine subsystem has also a unique solution. The result follows.

Let us consider situation when a sensor of turbocharger speed is not available. Assume that we have some prior knowledge about the compressor map (8) and it is as limited as: $I_{t c}=\left\{a_{8}=a_{8}^{\star}, a_{9}=0\right\}$. The rest of the coefficients of the compressor map are not fixed.

Proposition 7.2 The engine model (3)-(20) with the prior knowledge $I_{t c} \cup I_{a t}$ is globally identifiable for the parameter set $\mathcal{P}$ and the measurement set $\tilde{\mathcal{M}}_{i d} \backslash\left\{N_{t c}\right\}$ if the sign of parameter $a_{5}$ in (8) is known.

Proof of Proposition 7.3 Find the regressors dependent on turbocharger speed: $\phi_{\eta_{c}}^{t c}(t), \phi_{p c}^{t c}(t), \phi_{1}^{t c}(t)$. We obtain then four equations involving $N_{t c}$ and parameters:

$$
\begin{aligned}
y_{1}^{t c}(t) \eta_{c}(t) & =C_{p} w_{c}(t) y_{\eta_{c}}^{t c}(t) \\
y_{1}^{t c}(t) & =\theta_{1}^{t c} \phi_{1}^{t c}(t)+\theta_{2}^{t c} \phi_{2}^{t c}(t) \\
\eta_{c}(t) & =a_{4}+a_{5} N_{t c}(t)+a_{6} N_{t c}(t)^{2} \\
& +a_{7} w_{c}(t)+a_{8} w_{c}(t)^{2} \\
\phi_{1}^{t c}(t) & =-N_{t c}(t) \frac{\partial}{\partial t} N_{t c}(t)
\end{aligned}
$$

Denote $z_{1}(t)=C_{p} w_{c}(t) y_{\eta_{c}}^{t c}(t)$ and $z_{2}(t)=$ $a_{8} w_{c}(t)^{2}$. Form the command to Ritt's algorithm to generate input-output relationship eliminating $\eta_{c}(t), \phi_{1}^{t c}(t), N_{t c}(t)$. The resulting relationship, being a differential polynomial, contains many additive terms. Introducing canonical re-parameterization we get a system of algebraic equations. The number of new canonical parameters is much bigger then original ones: $\theta_{1}, \theta_{2}, a_{4} . a_{5}, a_{6}, a_{7}$. Using Grobner bases tools, the subsystem of equations was found

$$
\begin{aligned}
a_{6} \theta_{1} a_{7} & =b_{1} \\
a_{6} \theta_{1} & =b_{2} \\
a_{6} \theta_{1} \theta_{2} & =b_{3} \\
a_{6} a_{7}^{2} & =b_{4} \\
-a_{5}^{2} a_{6}+4 a_{6}^{2} & =b_{5}
\end{aligned}
$$

which is uniquely resolvable with respect to the original parameters if we know the sign of $a_{5}$ and there is no 
other equation in the full system resolvable with respect to $a_{5}$ without this knowledge. Referring to the section 4 the result follows.

In the text above the identifiability was treated in the structural sense without regarding the data trajectories. The formulation of persistent excitation conditions is straightforward according to the Section 4. We present these conditions in explicit form for the air throttle model.

Proposition 7.3 The persistent excitation condition for the air throttle model (10)- (11) are as follows:

$$
\frac{\partial}{\partial t} \alpha(t)\left(\frac{\partial}{\partial t} A_{a t}(t)\right)^{3} \neq 0
$$

for given input trajectories $\alpha(t), \mathcal{T}_{\text {load }}(t), w_{f}(t)$.

It is clear that these conditions are always satisfied if the throttle angle changes monotonically.

\section{Software issues and computational complexity}

In the work presented three programming packages for Maple computer algebra system were used. The basic program package is Ritt's algorithm implementation by [9] and its modification [4].

The main program rittio works in the following way:

$$
>\operatorname{rittio}([E],[Y],[X])
$$

where $E$ is the list of modeling equations, $Y$ is the list of ordered desirable variables, usually inputs, outputs and parameters, $X$ is the list of undesirable variables which are subject to elimination. If one specifies parameters as functions of time then the algorithm produces the complete characteristic set of a given DAE system. It requires many computations and at this time its computational limit is up to 10 time-dependent variables. If one specifies parameters as time-independent variables then the algorithm produces just input-output relationships. The computational limit increases significantly. In this way it was applied to the engine model given.

The second package is the convertor [4] from ODE to DAE systems. Its main program has two inputs:

$$
>\operatorname{ode} 2 a \operatorname{de}(L, t)
$$

the list of arbitrary ODE equations $L$ and independent variable $t$. It produces its differential-algebraic equivalent of minimal degree, automatically augmenting new variables if necessary. No computational problems have been noticed with this program.

The third package is the library of symbolic objects for modeling of internal combustion engines. This library, presented in [8], stores the basic structures usually used in modeling of internal combustion engines as black boxes. For example, the throttle symbolic model can be obtain by one line:

$$
>\text { orifice(inputs, outputs, parameters) }
$$

with the specification of variables and/or subscripts involved. These procedures are generated by the other procedures, containing the derivation of this model from the first principles. The first principles are also considered as black boxes in this library. Thus, some changes in the underlying physical assumptions automatically result in the modified model of a subsystem.

Additionally, we used the Grobner bases package included in the standard Maple set up.

The combination of these programs allows one to creatively automatize the engine modeling process.

\section{References}

[1] L. Ljung and T. Glad. On Global Identifiability for Arbitrary Model Parameterizations. Automatica, Vol. 30, No. 2, pp. 265-276, 1994.

[2] S. T. Glad, and L. Ljung. Identifiability with constraints. report. Linköping University, 1998.

[3] L. Ljung. System Identificataion. Prentice Hall, Englewood Cliffs, New Jersey, 1987

[4] P. Lindskog. Methods, Algorithms and Tools for System Identification Based on Prior Knowledge. PhD thesis 436, Department of Electrical Engineering, Linkoping University, Sweden, May 1996.

[5] M. Nyberg and L. Nielsen. Model Based Diagnosis for the Air Intake System of the SI Engine. $S A E$ technical paper 970209.

[6] J. B. Heywood. Internal Combustion Engine Fundamentals. 1988.

[7] A. Hendriks and S. Sorenson. Mean value modeling of spark ignition engines, SAE Technical paper 900616 .

[8] A. Sokolov, A. Loria. An approach to structural modeling of nonlinear systems facilitating robust design: application to IC engines. MTNS'98, July, Padova, 1998.

[9] S. T. Glad. Implementing Ritt's algorithm of differential algebra. In IFAC Symposium on Control Systems Design, NOLCOS'92, pages 610-614, Bordeaux, France, June 1992.

[10] S. T. Glad. Nonlinear input-output relations and identifiability. In Proc. 31st $C D C$, Tucson, December 1992.

[11] A. Sokolov Generalized model of flow restrictions for IC engine control applications. Technical report N2120, 1999, ISY, Linköping University.

[12] A. Sokolov, T. Glad Identifiability of Turbocharged IC Engine Models SAE Technical paper N1999-01-0216 